\title{
Toxic Substances in Cancer
}

\section{Effects on the Ciliary Movement of the Oyster Gill}

By

\author{
Iwao Yamaguchi and Kiyota Oh-Uti \\ （山口 䈆）（大内清太）
}

From Prof. M. Muto's Surgical Clinic, Tohoku University, Sendai

(Received for publication, July 1, 1957)

In a work of a series of chemical studies on cancer undertaken by the senior author (K. O.) and his coworkers in our clinic, Makil demonstrated that the extract of gastric carcinoma showed a definitely higher inhibition to the pulsation of an isolated frog heart than the extract of normal gastric mucosa, and ascribed this inhibition to substances passing through a cellophane membrane. Then, Hasegawa ${ }^{21}$ and $\mathrm{Abe}^{31}$ investigated these substances in various aspects.

With a view to obtaining further information as to their nature, we caught them by the use of ion exchangers, fractionated by steam distillation and by the use of organic solvents, and tested the fractions for effects on the mechanical activity of cilia of the oyster gill by the modified Nomura and Tomita method ${ }^{4}$. The results of this investigation form the subject of the present paper.

We wish to express our sincere thanks to Assistant Professor G. Tomita of the Biological Institute of our university for his constant advice.

\section{EXPERIMENTAI}

\section{Preparation of the Test Material}

The material studied in this work consisted of extracts of dried tissues of gastric carcinoma and normal gastric mucosa. First, a specimen of gastric carcinoma or normal gastric mucosa, which was obtained at operation in our clinic, was soon washed in water to remove blood, mucus, and other foreign substances, wiped with pieces of gauze to remove water, cut into fine pieces, vacuum-dried in a desiccator over sulfuric acid to a mummy, ground to fine powder, and stored until used as extraction material.

\section{Method of Testing the Material for Ciliary Inhibition}

The oyster (Ostrea gigas) cultured in Matsushima Bay was used. After carefully removing the right (upper) valve and a part of the mantle, a band of uniform width (3-4 mm.) was cut out from the ventral margin of a gill plate 
and left in sea water for several hours, where it would fully recover from the shrinkage due to mechanical stimulations at cutting. The band was then divided into nearly rectangular pieces by cutting along ventral ridges at equal intervals of about $3 \mathrm{~mm}$. The "gill pieces" thus obtained will "crawl" on the bottom of the container in the direction shown by an arrow in Figure 1, owing to the reaction against the beating of water by the cilia on their surface. All the gill pieces obtained from an individual oyster ( 80 to 140 in total) were pooled and left in sea water.

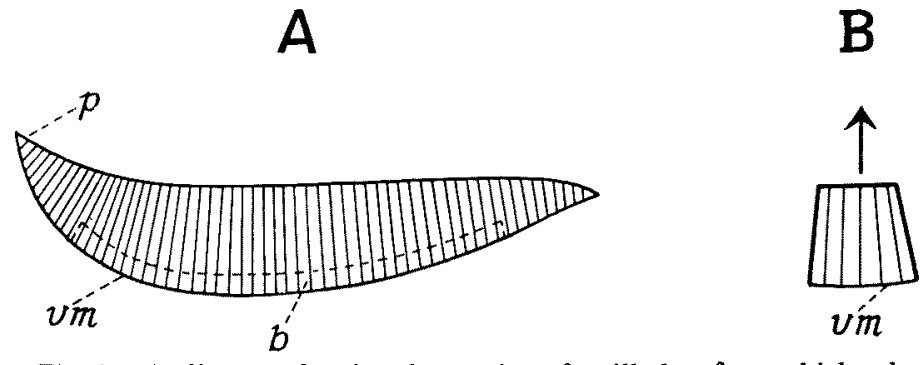

Fig. 1. A, diagram showing the portion of a gill plate from which a band was cut to prepare "gill pieces." B, diagram showing a "gill pieces," with its direction of advance indicated by an arrow. $b$, band; $p$, posterior end ; vm, free ventral margin.

The sea water to be used for experiments was obtained from Matsushima Bay and filtered before use, which showed a $\mathrm{pH}$ of 7.8 .

In making a test, 10 gill pieces were placed in a small flat-bottomed Petri dish containing normal sea water. With a stop watch graduated in 1/10 second divisions and a magnifier $(\times 4-5)$, the time required for each gill piece to advance $1 \mathrm{~mm}$. was measured, and the speed of the gill piece $(\mathrm{mm} . / 10 \mathrm{sec}$.) was calculated. The mean of the speeds of the 10 gill pieces $(8.2-12.6)$ was then calculated and taken to be the speed before experiment. The sea water in the Petri dish was then replaced by the solution to be tested, and the speeds of the gill pieces were measured after 1, 2, and 3 hours. The percentage of the mean speed before experiment was computed and taken to denote the relative mechanical activity of cilia at that time under the given experimental conditions.

\section{Tests of Aqueous Extracts on Oyster Gill Pieces}

1) Powdered aqueous extracts. Gastric carcinoma or normal gastric mucosa was extracted at room temperature (lower than $10^{\circ} \mathrm{C}$.) with 15 times as much distilled water with constant shaking for 24 hours. The extract thus obtained was filtered and centrifuged. The supernatant liquid was concentrated under reduced pressure to a syrup and dried in a desiccator to powder. Thirty mg. of this powder dissolved almost completely in $15 \mathrm{cc}$. of sea water, giving no sediment after centrifugation. Test solutions were prepared by doubling dilution of this stock solution (a 1:500 solution of the powdered aqueous extract).

As shown in Figure 2, the extract of gastric carcinoma (hereafter to be 

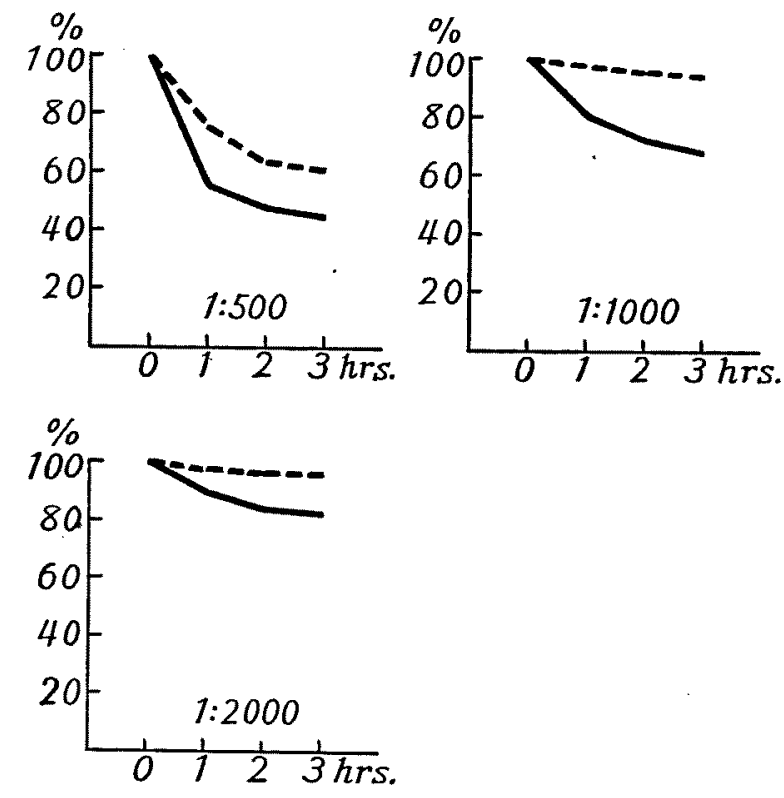

Fig. 2. Aqueous extract. - Gastric carcinoma. - - Normal gastric mucosa. O means "just before experiment."

referred to simply as "carcinoma extract") inhibited ciliary movement remarkably at dilutions up to $1: 1000$ and slightly at $1: 2000$, whereas the ciliary inhibition of the normal mucosa extract was weaker than that of the carcinoma extract (remarkable at 1:500, but only slight at $1: 1000$, and invisible at $1: 2000$ ).

2) Experiments of dialysis. An aqueous extract was concentrated under reduced pressure to one third of the initial volume, placed in a cellophane bag, and dialyzed against distilled water for 48 hours in an ice chamber, during which time the dialyzate (outer liquid) was replaced by distilled water every 8 hours. The dialyzates were pooled. The combined dialyzate and the "inner liquid" (48-hour-dialyzed extract) were concentrated under reduced pressure and dried in a desiccator to powder.

Both the inner liquid of the carcinoma extract and that of normal mucosa extract showed only a slight ciliary inhibition at a dilution of $1: 500$, and no inhibition at $1: 1000$. On the other hand, the dialyzates of both extracts inhibited ciliary movement more markedly than the inner liquids, and especially the dialyzate of the carcinoma extract showed a remarkable inhibition at dilutions up to $1: 2000$, and a slight inhibition at $1: 4000$ (the inhibition shown by the dialyzate of normal mucosa extract being remarkable only at $1: 500$, slight at $1: 1000$, and invisible at 1:2000) (Fig. 3).

\section{Tests of a Sea-water Extract of Gastric Carcinoma on Oyster Gill Pieces}

The dry powder of gastric carcinoma was extracted with 15 times as much 

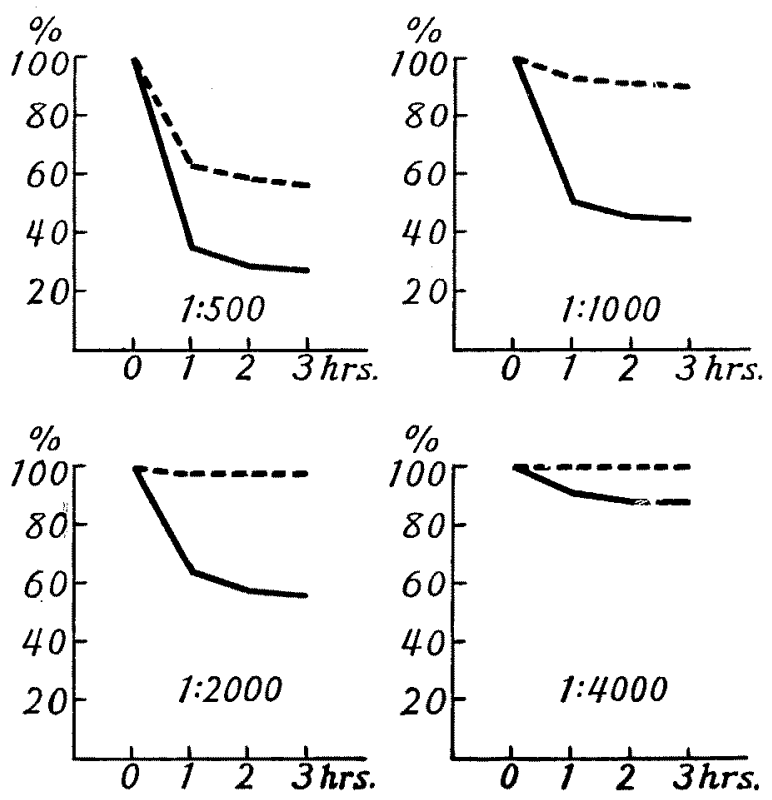

Fig. 3. Dialyzates.

Gastric carcinoma. --- Normal gastric mucosa.

Fig. 4

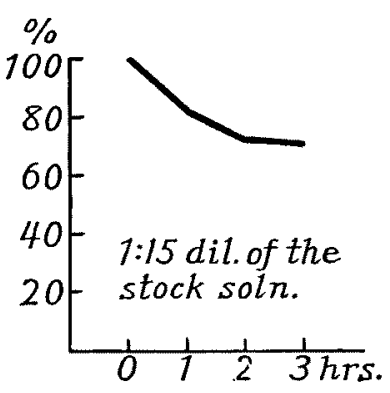

Fig. 5

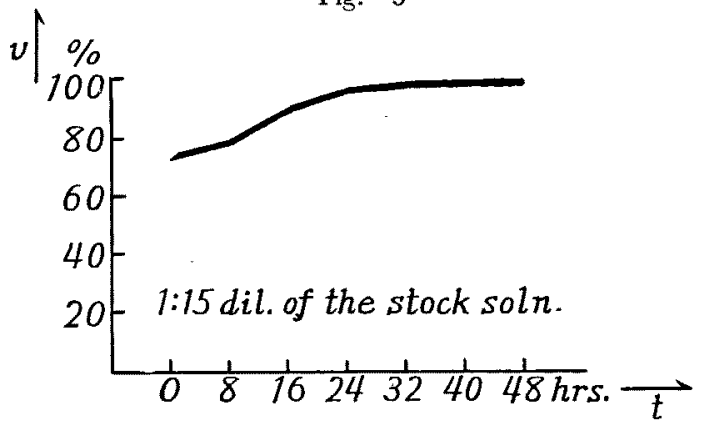

Fig. 4. Sea-water extract of gastric carcinoma.

Fig. 5. Dialysis of the sea-water extract of gastric carcinoma. v, activity at the end of two hours' exposure. $t$, time elapsed after the start of dialysis.

sea water. This extract (stock solution of the carcinoma extract) was diluted with sea water 15 -fold. Tests of this dilute solution for ciliary inhibition gave positive results as shown in Fig. 4. Then, the stock solution of the carcinoma extract was dialyzed through a cellophane memarane against sea water. In the course of dialysis, samples of the inner liquid were taken at intervals of 8 hours, diluted with sea water 15-fold, and tested for ciliary inhibition. As shown in Figure 5, it was found that the test solution had lost its power of ciliary inhibition almost entirely through 24 hours' dialysis. 
V. Experiments on the Dialyzate of the Gastric Carcinoma Extract

As described above, the preliminary tests showed that the substances responsible for the ciliary inhibition of the carcinoma extract would pass through a cellophane membrane almost entirely within 24 hours. In order to study their nature, several tests of chemical and physical treatments and of systematic fractionation were made on the dialyzate of the carcinoma extract (hereafter to be referred to simply as "carcinoma dialyzate").

1) Effect of heating. Heating at $100^{\circ} \mathrm{C}$ under reflux for 20 minutes had almost no effect on the power of ciliary inlibition of a 1:1000 dilution of the carcinoma dialyzate (Fig. 6).

2) Effect of incineration. Thirty $\mathrm{mg}$. of the powdered carcinoma dialyzate was fully incinerated in a porcelain crucible. The gray-white powder obtain (9.7 mg.) was dissolved in a small amount of $\mathrm{N} / 10 \mathrm{HCl}$, to which was added sea water to make up to $15 \mathrm{cc}$. The solution was filtered, and tested on gill pieces after adjustment of the $\mathrm{pH}$ to 7.8 with $\mathrm{N} / 10 \mathrm{NaOH}$. It was found that the test solution (corresponding to a 1:500 dilution of the carcinoma dialyzate) showed no ciliary inhibition (Fig. 7).
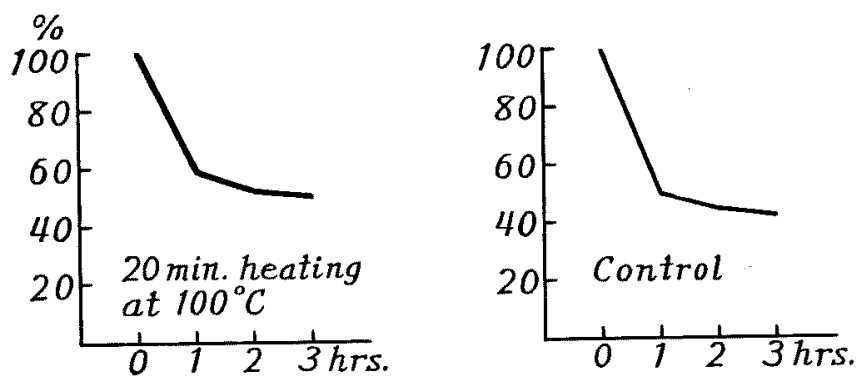

Fig. 6. Effect of heating carcinoma dialyzate. Control, 1: 1000 dilution of carcinoma dialyzate.
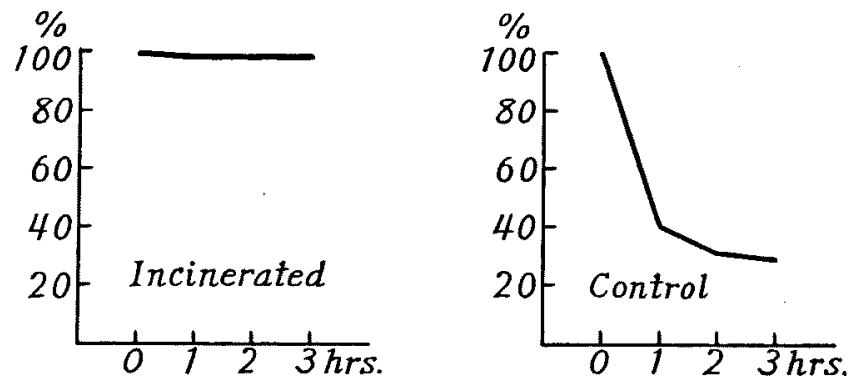

Fig. 7. Effect of incineration of carcinoma dialyzate. Control, 1:500 dilution of carcinoma dialyzate.

3) Tests of solubility in organic solvents. Aliquots of $15 \mathrm{mg}$. of the powdered carcinoma dialyzate were extracted under reflux with boiling organic solvents. 
Each extract was dried to powder by evaporation of the solvent. The residue was dried to powder after being washed with the solvent. Each preparation of powder was dissolved in $15 \mathrm{cc}$. of sea water (corresponding to a 1:1000 dilution of the carcinoma dialyzate), and tested for ciliary inhibition after having its $\mathrm{pH}$ adjusted to 7.8. Thus, it was found that the substances responsible for the ciliary inhibition of the carcinoma dialyzate were highly soluble in ethanol, fairly soluble in amyl alcohol and chloroform, but almost insoluble in ether and acetone (Fig. 8).
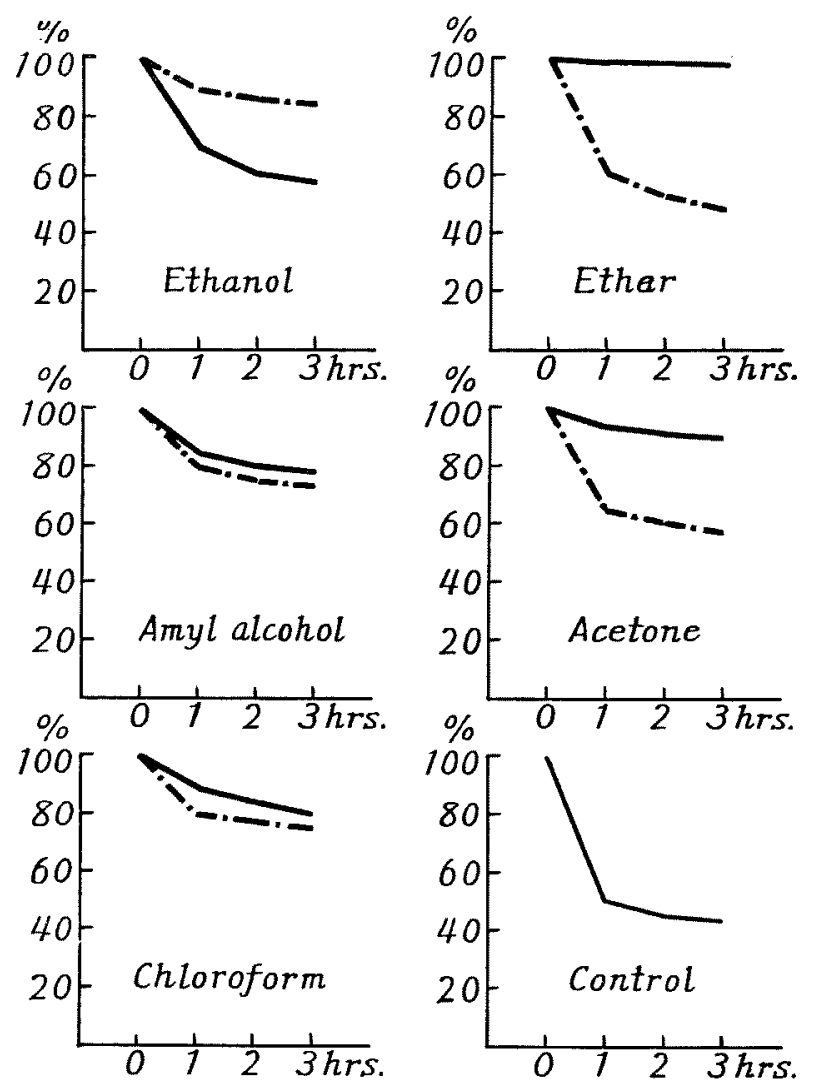

Fig. 8. Solubility of effective substances in various organic solvents. —- extract, - - - residue, Control, 1:1000 dilution of carcinoma dialyzate.

4) Effect of treating with trichloroacetic acid. When an equal volume of $20 \%$ trichloroacetic acid was added to an aqueous solution containing $15 \mathrm{mg}$. of the powdered carcinoma dialyzate, there appeared a slight degree of white turbidity. The mixture was centrifuged. The supernatant liquid was neutralized, concentrated under reduced pressure, and dried. This dry material was dissolved in $15 \mathrm{cc}$. of sea water, and the $\mathrm{pH}$ of the solution was adjusted to 7.8. The sediment was washed with $10 \%$ trichloroacetic acid, dissolved in a small amount 
of $\mathrm{NaOH}$-alkalized sea water. The solution was diluted to $15 \mathrm{cc}$. with sea water, and tested on gill pieces after adjustment of the $\mathrm{pH}$ to 7.8 .

In tests of these solutions on gill pieces, the "supernatant" solution inhibited ciliary movement much more remarkably than the "sediment" solution, the inhibition being only a little weaker than a $1: 1000$ dilution of the untreated carcinoma dialyzate (Fig. 9).
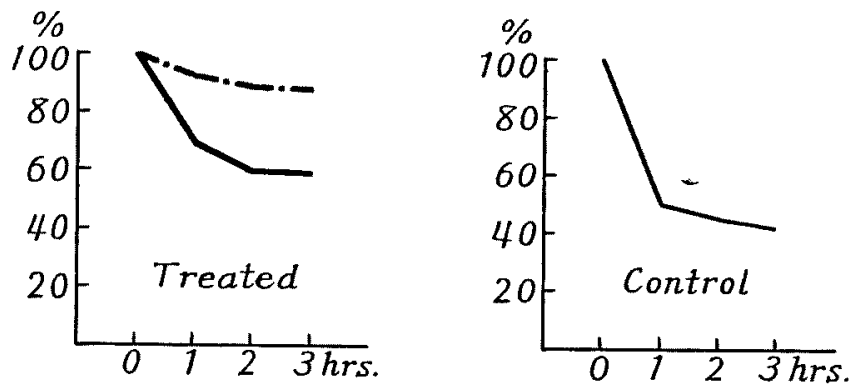

Fig. 9. Effect of treating carcinoma dialyzate with trichloroacetic acid. _ supernatant, - - - precipitate after trichloroacetic acid treatment. Control, $1: 1000$ dilution of carcinoma dialyzate.

5) Effect of treating with phosphotungstic acid. To an aqueous solution containing $15 \mathrm{mg}$. of the powdered carcinoma dialyzate was added a small excess of a saturated aqueous solution of phosphotungstic acid. A great amount of precipitate produced was set aside by centrifugation after 24 hours. Excess phosphotungstic acid in the supernatant liquid was removed by addition of barium hydroxide. The barium was then removed with carbon dioxide, and the supernatant liquid was concentrated to powder. This "supernatant." powder was dissolved in $15 \mathrm{cc}$. of sea water (corresponding to a 1:1000 dilution of the carcinoma dialyzate), and was tested on gill pieces after adjustment of the $\mathrm{pH}$ to 7.8. The ciliary inhibition shown by the solution was only slight, however (Fig. 10).
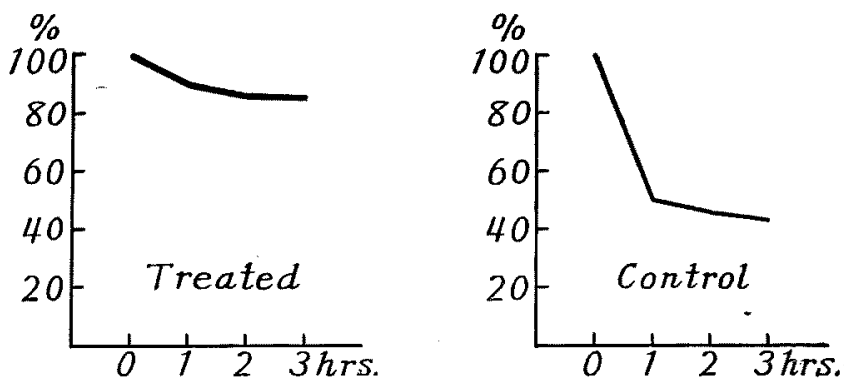

Fig. 10. Effect of treating carcinoma dialyzate with phosphotungstic acid. Treated, supernatant liquid after phosphotungstic treatment. Control, 1: 1000 dilution of carcinoma dialyzate. 
6) Effect of acid hydrolysis. To $15 \mathrm{mg}$. of the powdered carcinoma dialyzate was added $3 \mathrm{cc}$. of $6 \mathrm{~N} \mathrm{HCl}$, and the mixture was boiled under reflux for 12 hours. The resulting black substance was removed by filtration through filter paper after cooling. The filtrate was concentrated under reduced pressure to dryness. The dry material was dissolved in $5 \mathrm{cc}$. of distilled water, and the solution was concentrated to dryness. After removal of most of the $\mathrm{HCl}$ by repeating the treatment three times, the material was dissolved in $15 \mathrm{cc}$. of sea water (corresponding to a $1: 1000$ dilution of the carcinoma dialyzate), and tested on gill pieces after adjustment of the $\mathrm{pH}$ to 7.8 . It was found that the power of ciliary inhibition of this solution was about half that of a $1: 1000$ dilution of the carcinoma dialyzate (Fig. 11).
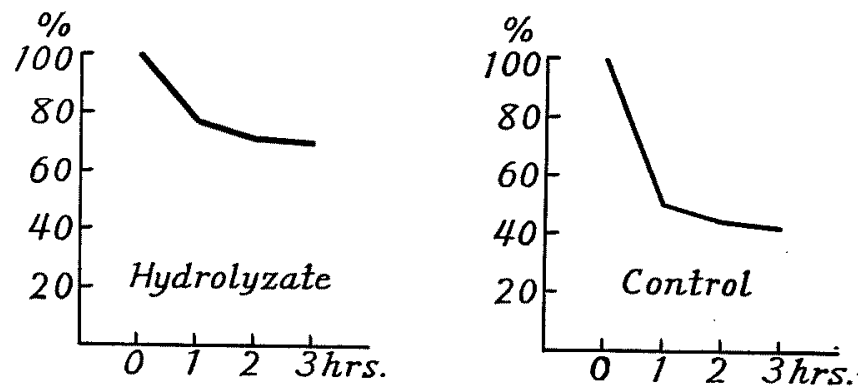

Fig. 11. Effect of acid hydrolysis. Control, $1: 1000$ dilution of carcinoma dialyzate.

7) Effect of treating with adsorbents. An aqueous solution containing $15 \mathrm{mg}$. of the powdered carcinoma dialyzate was acidified with $\mathrm{HCl}$ to $\mathrm{pH} 4.5$, treated with $1 \mathrm{~g}$. of kaolin or $0.5 \mathrm{~g}$. of powdered blood charcoal, and filtered through filter paper. The filtrate was concentrated under reduced pressure to dryness. The dry material was dissolved in $15 \mathrm{cc}$. of sea water, the $\mathrm{pH}$ of which was adjusted to 7.8. The residue, together with the filter paper, was extracted at $40^{\circ} \mathrm{C}$. in $\mathrm{NaOH}$-alkalized distilled water $(\mathrm{pH} \mathrm{8)}$, and the extract was filtered. The filtrate was neutralized and concentrated to dryness. The dry material was dissolved in $15 \mathrm{cc}$. of sea water (corresponding to a 1:1000 dilution of the carcinoma dialyzate), and the $\mathrm{pH}$ was adjusted to 7.8. It was found that both the unadsorbed part and the adsorbed and redissolved part of the carcinoma dialyzate showed ciliary inhibition, but the former was more effective (Fig. 12).

8) Effect of passing through ion exchangers. An anion exchanger of ammonium type, Aberlite IR A-410 (column height: $15 \mathrm{~cm}$.) was well washed first with $2 N \mathrm{NaOH}$, then with water. An amount of the carcinoma dialyzate was passed through the column and the effluent was neutralized and concentrated under reduced pressure to dry powder. An amount of the carcinoma dialyzate was passed through a cation exchanger of sulfonic acid type, Amberlite IR 120 (column height : $15 \mathrm{~cm}$.), which had previously been treated with $2 \mathrm{~N} \mathrm{HCl}$ and washed with water, and dry powder was obtained in the same manner as before. Each preparation of powder was dissolved in sea water to obtain a 1:1000 dilu- 

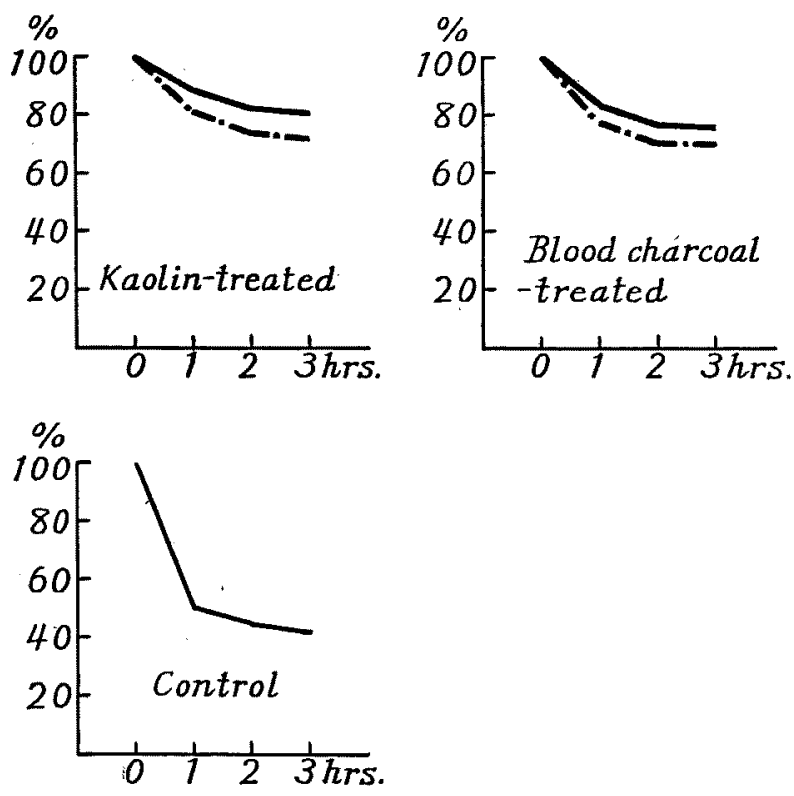

Fig. 12. Effect of adsorbents on carcinoma dialyzate. - adsorbed, - - - unadsorbed. Control, 1:1000 dilution of carcinoma dialyzate.
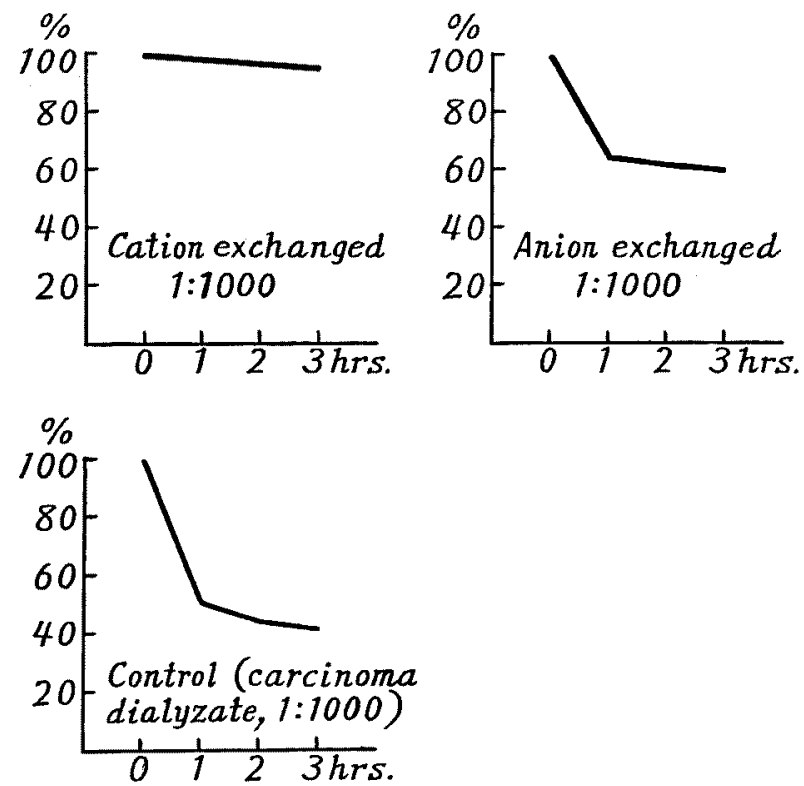

Fig. 13. Effect of passing through ion exchangers on carcinoma dialyzate. 
tion, the $\mathrm{pH}$ of which was adjusted to 7.8 .

In tests of these two solutions on gill pieces, the ciliary inhibition of the anion-exchange effluent was only a little weaker than a $1: 1000$ dilution of the carcinoma dialyzate, whereas the cation-exchange effluent showed no inhibition (Fig. 13).

9) Systematic fractionation. Fractionation was performed by reference to the method of Satake ${ }^{5}$ (Table 1). Thus, a cation exchanger of carboxylic acid type, Amberlite IR C-50 (column height: $15 \mathrm{~cm}$.) was well washed first with $2 \mathrm{~N} \mathrm{NaOH}$, then with water, and the $\mathrm{pH}$ was adjusted to 5 with acetic acidsodium acetate buffer. A concentrated carcinoma dialyzate ( 1 litter being concentrated to $80 \mathrm{cc}$.) was passed through the column at the rate of $2 \mathrm{cc}$. per minute. The column was then washed with $100 \mathrm{cc}$. of distilled water. The effluent and the washings were combined and concentrated under reduced pressure to dryness (fraction 1). Then, $40 \mathrm{cc}$. of $1 \mathrm{~N} \mathrm{HCl}$ was passed through the column at a rate of $1 \mathrm{cc}$. per minute, followed by $20 \mathrm{cc}$. of distilled water. The two effluents were combined, alkalized by addition of $15 \mathrm{cc}$. of $20 \% \mathrm{NaOH}$, and soon subjected to steam distillation. The distilled vapor and liquid were received in $5 \mathrm{cc}$. of $1 \mathrm{~N} \mathrm{HCl}$. The distillation was stopped when the distillate attained to a volume of $25 \mathrm{cc}$. The distillate was extracted with $30 \mathrm{cc}$. of ether three times, and the extract and the residue were evaporated to dryness (fractions 2 and 3 ). The residue of the steam distillation was extracted with constant

\section{TABLE I}

Scheme for Fractionation of Carcinoma Dialyzate

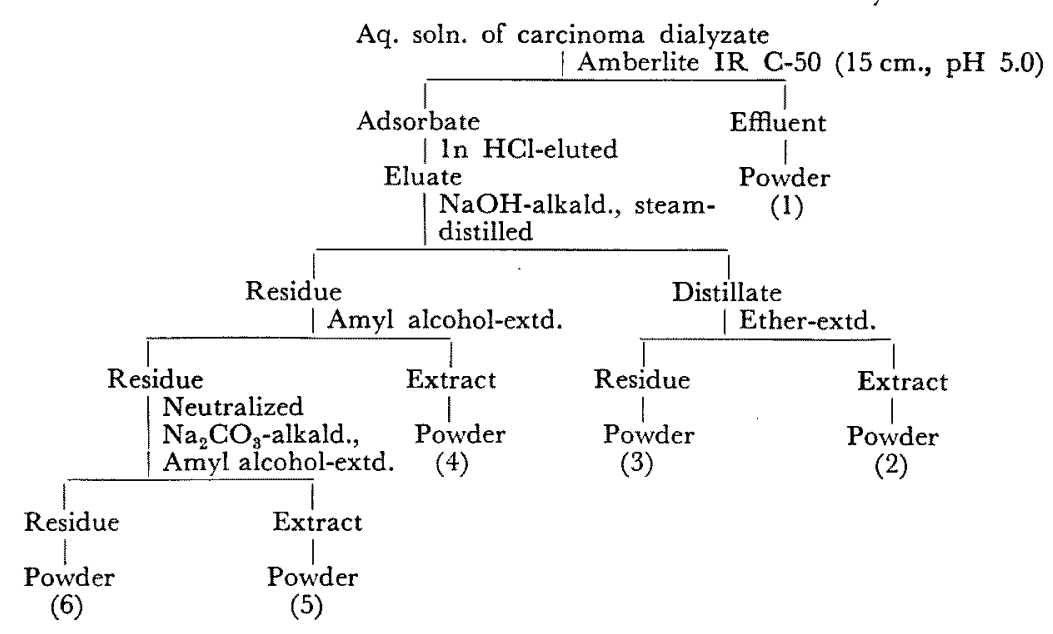

shaking with $40 \mathrm{cc}$. of amyl alcohol. The amyl alcohol layer was further extracted with constant shaking with $30 \mathrm{cc}$. of $\mathrm{N} / 2 \mathrm{HCl}$, and the dilute- $\mathrm{HCl}$ layer was evaporated to dryness (fraction 4). The residue of the amyl alcohol extraction was neutralized with $\mathrm{HCl}$, alkalized with sodium carbonate, and extracted again with $40 \mathrm{cc}$. of amyl alcohol. The new amyl alcohol layer was 
extracted with $30 \mathrm{cc}$. of $\mathrm{N} / 2 \mathrm{HCl}$ as before, and the dilute-HCl layer was evaporated to dryness (fraction 5). The carbonate-alkaline residue was neutralized with $\mathrm{HCl}$ and concentrated under reduced pressure to dryness (fraction 6). Each of the six fractions thus obtained was dissolved in sea water and tested on gill pieces.

Fraction 1 showed some degree of ciliary inhibition at a dilution of $1: 500$, but the inhibition was much weaker than that of the same dilution of the carcinoma dialyzate, and it showed no inhibition at 1:1000. Fraction 2 (ether soluble fraction of the distillate of steam distillation) was found to inhibit ciliary movement only slightly when tested at $1: 1000$, while fraction 3 (ether-insoluble fraction) showed a remarkable inhibition even at 1:2000. Of all the six fractions, fraction 4 (amyl alcohol-soluble fraction of the distillation residue) was
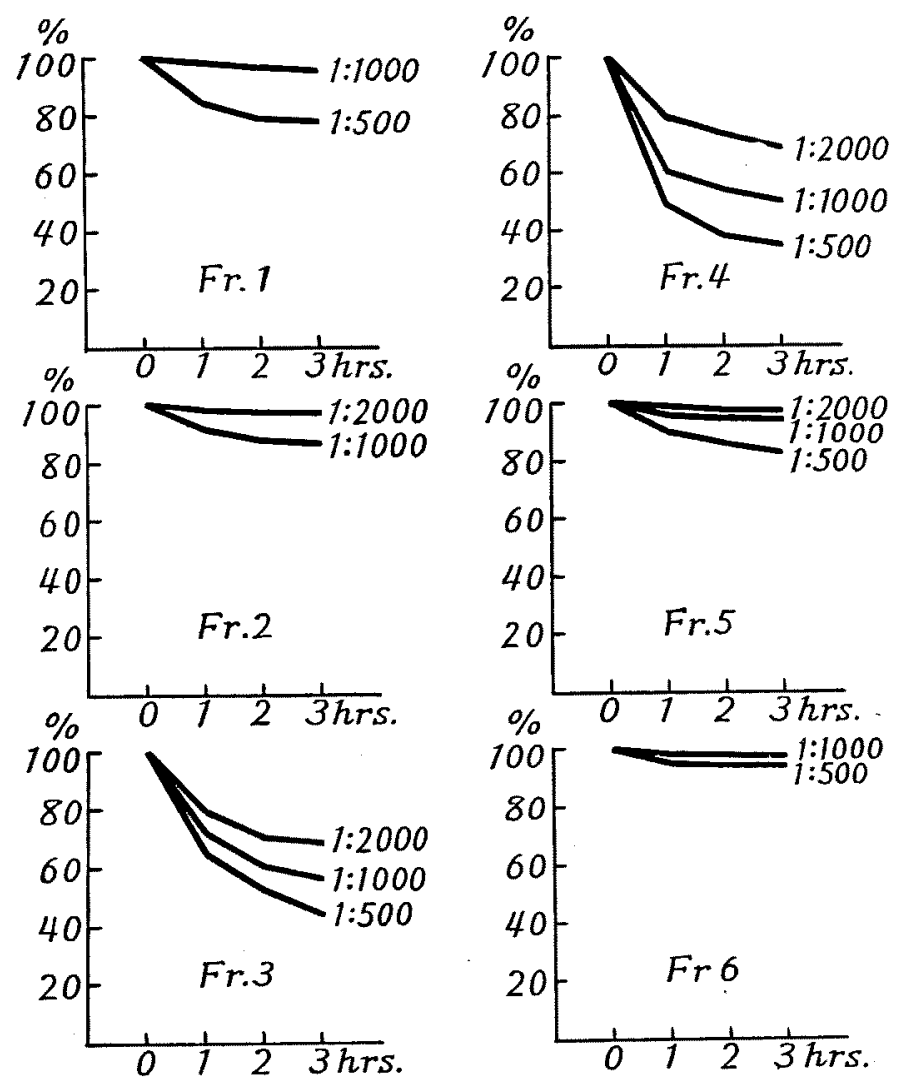

Fig. 14. Fractions of carcinoma dialyzate.

found to have the strongest inhibitory effect on ciliary movement, the inhibition being definite even at 1:2000. The ciliary inhibition shown by fraction 5 (amyl alcohol-soluble fraction obtained from the $\mathrm{Na}_{2} \mathrm{CO}_{3}$-alkalized residue) was 
quite weak at 1:500, while fraction 6 showed no inhibition at the same dilution (Fig. 14).

\section{Discussion and Conclusions}

In our tests on gill pieces of the oyster, both the aqueous extract of gastric carcinoma and that of normal gastric mucosa had inhibitory effects on the ciliary movement, but the ciliary inhibition of the former was definitely stronger than that of the latter. These extracts lost their power of ciliary inhibition through dialysis. Thus, the powder prepared from a 48 hours' dialyzate (liquid outside a cellophane bag) showed much stronger ciliary inhibition than the powder of the inner liquid.

In order to ascertain whether the substances responsible for the ciliary inhibition shown by the carcinoma dialyzate were identical with the substances that were found by Maki to inhibit an isolated frog heart, effects of various physical and chemical treatments on the ciliary inhibition of the powdered carcinoma dialyzate were investigated. From the results obtained it may be concluded that there are no noteworthy differences between the two groups of effective substances. Thus, substances inhibiting the ciliary movement of the oyster gill were weakened by 20 minutes' heating at $100^{\circ} \mathrm{C}$., completely destroyed by incineration, very soluble in ethanol, to some extent soluble in amyl alcohol and chloroform if boiled, but almost insoluble in acetone and ether. Further, they were remarkably weakened in effectiveness by passage through a cation exchanger, but not much affected by passage through an anion exchanger.

In addition, it was found that the substances did not entirely lose their inhibitory action by acid hydrolysis, and were precipitated little with $10 \%$ trichloroacetic acid but fully with phosphotungstic acid.

From these findings, especially of their behavior to dialysis and ion exchange, it was inferred that the substances inhibiting the ciliary movement of the oyster gill were possibly basic ones of small molecular weight. With a view to isolation of these substances, attempts were then made to fractionate them by a combination of ion exchange, steam distillation, and extraction with organic solvents. From the results described in the previous section it may be concluded that the substances responsible for the inhibitory effect of gastric carcinoma extracts on the ciliary movement of the oyster gill, consist at least two basic compounds of small molecular weight, volatile and non-volatile.

\section{SUMMARY}

1. Aqueous extracts of both gastric carcinoma and normal gastric mucosa inhibited the mechanical activity of gill cilia of the oyster, and the 
ciliary inhibition of the former was definitely stronger than that of the latter.

2. The substances responsible for the ciliary inhibition passed through a cellophane membrane almost entirely in 24 hours of dialysis against sea water.

\section{References}

1) Maki, Tohoku J. Exp. Med., 1956, 63, 121.

2) Hasegawa, Tohoku Igaku Zasshi, (Jap.), 1955, 52, 6.

3) Abe, ibid., in press.

4) Tomita, Sci. Rep. Tohoku Univ., 4th Ser, 1955, 21, 1.

5) Satake, Standard methods for biochemical experiments (Jap.), Tokyo, 1953, p. 78. 